\title{
Modeling and Characterization of Damage Processes in Metallic Materials
}

\author{
E.H. Glaessgen ${ }^{*}$, E. Saether ${ }^{\dagger}$, S.W. Smith, and J.D. Hochhalter ${ }^{\dagger}$ \\ Durability, Damage Tolerance and Reliability Branch \\ NASA Langley Research Center, Hampton, VA, 23681 \\ and \\ V.I. Yamakov and V. Gupta \\ National Institute of Aerospace, Hampton, VA, 23666
}

\begin{abstract}
This paper describes a broad effort that is aimed at understanding the fundamental mechanisms of crack growth and using that understanding as a basis for designing materials and enabling predictions of fracture in materials and structures that have small characteristic dimensions. This area of research, herein referred to as Damage Science, emphasizes the length scale regimes of the nanoscale and the microscale for which analysis and characterization tools are being developed to predict the formation, propagation, and interaction of fundamental damage mechanisms. Examination of nanoscale processes requires atomistic and discrete dislocation plasticity simulations, while microscale processes can be examined using strain gradient plasticity, crystal plasticity and microstructure modeling methods. Concurrent and sequential multiscale modeling methods are being developed to analytically bridge between these length scales. Experimental methods for characterization and quantification of near-crack tip damage are also being developed. This paper focuses on several new methodologies in these areas and their application to understanding damage processes in polycrystalline metals. On-going and potential applications are also discussed.
\end{abstract}

\section{Introduction}

Metallic materials are often considered by engineers to be homogeneous and isotropic bodies that can be characterized using the paradigm of continuum mechanics. In this definition, homogeneity implies that the mechanical and other properties of the material exhibit no spatial variation while isotropy implies that the mechanical and other properties of the material are identical regardless of geometric orientation or viewpoint. These approximations are implicit in many engineering analyses and are the basis for much of the work in the field of engineering fracture mechanics.

Engineering fracture mechanics relies on the comparison between computed fracture metrics and their empirically determined critical values. The paradigm involves asserting a fracture parameter, experimentally determining critical values of the parameter using coupon tests, and predicting crack growth in a structure by comparing computed metrics based on the parameter to the critical values. Thus, all fracture mechanics-based predictions of crack growth rely on the (implicitly) assumed similitude between the conditions under which the fracture parameters were determined and the operating conditions of the subject structure. Examples of these conditions include loading mode mixity, material thickness and operating environment. Although this paradigm is widely used for modeling crack growth at structural scales, it does not describe the fundamental processes that govern fracture. As a result, deviation from the conditions under which the critical fracture parameters were determined weakens the assumed similitude and may result in unforeseen consequences including unexpected failures.

\footnotetext{
*AIAA Associate Fellow

†AIAA Member
} 
Typically, the smaller the length scales involved, the less valid are the implicit approximations made using engineering fracture mechanics. The development of materials and structures such as nanocrystalline metals, microelectromechanical devices (MEMS), gradient microstructures and other emerging material and structural configurations may be highly dependent on developing an understanding of internal damage processes at nano-tomicro length scales (Needleman, 2001). Figure 1 depicts representative microstructures of a traditional aluminum alloy and a nanocrystalline metal in addition to a high-magnification image of a failed MEMS device. Note the two orders of magnitude difference between the respective length scale markers and the multiple order of magnitude difference between characteristic features. Although each of these configurations is very different, crack initiation and growth in each is dominated by events and processes occurring at length scales on the order of nanometers to microns that cannot be understood, predicted or characterized using standard engineering methods.

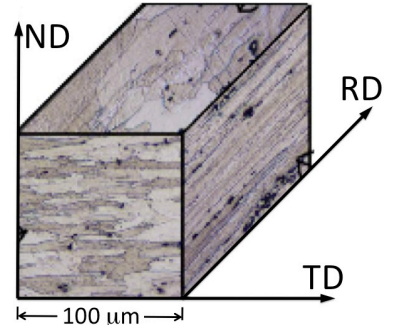

Aluminum 7075-T651

(Emery, 2009)

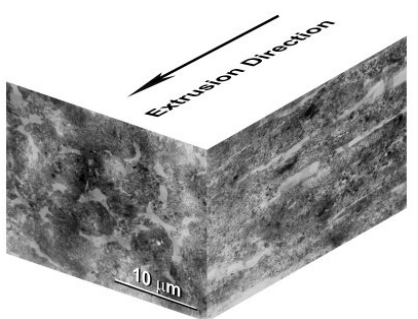

Nanocrystalline Al-Ti-Cu Alloy

(Lee, 2002)

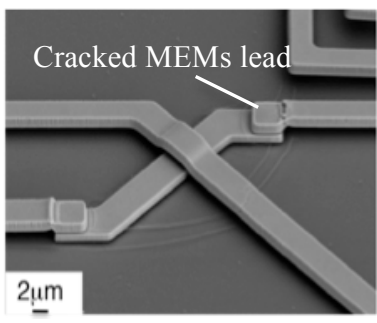

Fracture in MEMS device

(D'Agostino, 2005)

Figure 1: Examples materials and devices having small characteristic dimensions (note the length scale markers)

This paper describes a broad effort that is aimed at understanding the fundamental mechanisms of crack growth and using that understanding as a basis for designing materials and enabling predictions of fracture in materials and structures that have small characteristic dimensions. Much of the work discussed herein has been performed by the authors, or by the affiliated collaborators mentioned in the acknowledgements, and noted in the references; however, important external references are also included to provide additional perspective. The paper consists of a high-level overview, a discussion of some recent computational and experimental developments, and a few concluding remarks.

\section{Damage Science - Understanding Damage Processes At Nano-to-Micro Length Scales}

Thorough examination of damage processes must be undertaken at length scales at which a material cannot be considered to be a continuum (Raabe, 1998; Van der Giessen, 2002). Here, it is convenient to define two generalized length scales over which damage processes may be characterized - the nanoscale (approximately $10^{-9}$ to $10^{-6} \mathrm{~m}$ ) and the microscale (approximately $10^{-6}$ to $10^{-3} \mathrm{~m}$ ). From a viewpoint taken at the nanoscale, the most fundamental damage processes including free surface creation and dislocation formation, interaction and evolution are considered. From the viewpoint of the microscale, the development of nanoscale damage mechanisms progresses into aggregated processes such as small crack formation and plastic gradients near crack tips. Additionally, an intermediate length scale, the mesoscale, is sometimes used to indicate characteristic dimensions and processes at the upper end of the nanoscale or the lower end of the microscale. Damage Science focuses on the development of analysis and characterization methods needed to predict the formation, propagation, and interaction of fundamental damage mechanisms across these length scales.

Figure 2 presents the characteristic dimensions at which damage may be characterized, the associated physical features and some corresponding methods that may be employed. At the nanoscale, methods such as molecular dynamics are used to determine the most fundamental damage processes. At the mesoscale, methods such as dislocation dynamics are used to model the generation, motion, accumulation and annihilation of relatively large numbers of dislocations within a grain. At the microscale, various small-scale continuum plasticity formulations, cohesive zone models and finite element analyses are used to describe damage processes within individual grains or within polycrystals. Because no single analysis or experimental characterization approach can describe all of the relevant physics of crack growth, approaches that are developed at each length scale should be integrated within a 
rigorous multiscale modeling framework that spans some or all of these length scales. Additionally, in-situ experimentation is needed to aid in understanding the complexities of damage under realistic conditions, help to validate the nano/micro-scale computational results and provide input for the microscale analyses. The following text is a discussion of work in each of these areas.

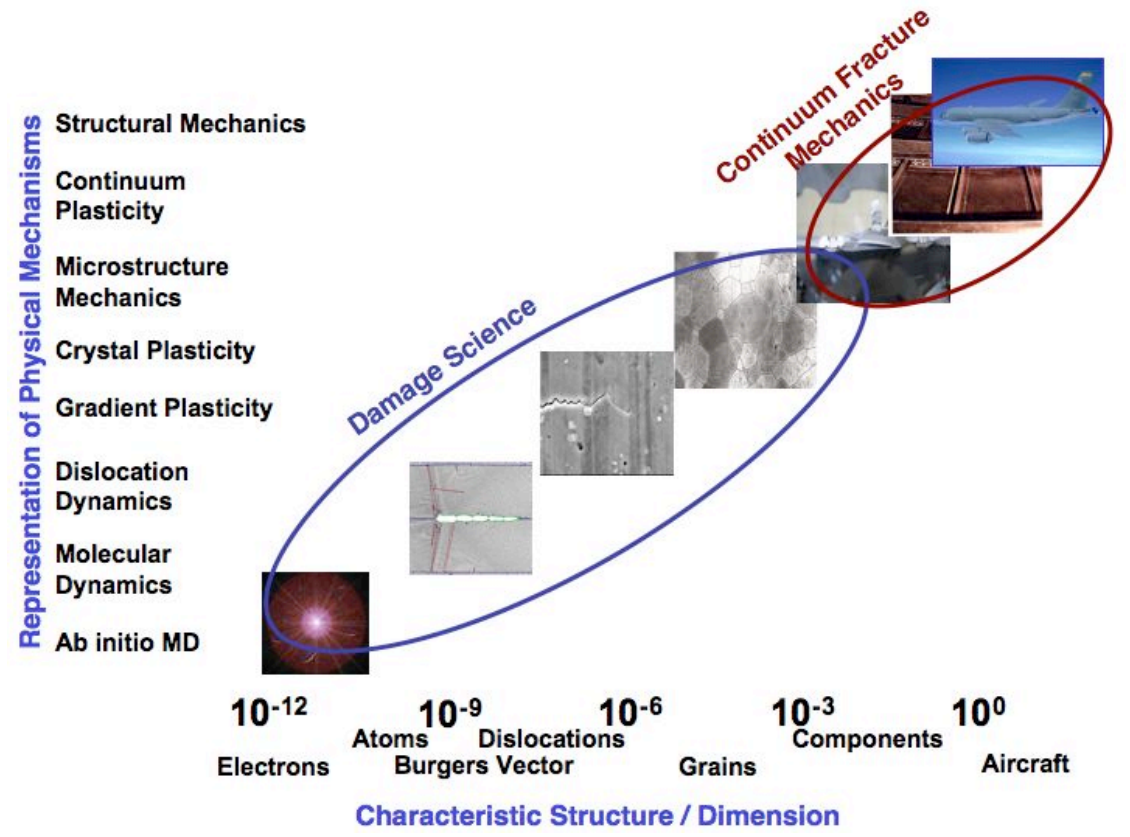

Figure 2: Characteristic dimensions and associated simulation methods

\section{A. Atomistic Simulation}

The most exacting interrogation of the fundamental interactions between atoms, including interactions that are near a crack tip, is performed via quantum mechanical analysis (Nair, 2010). However, quantum mechanical solutions for the interatomic forces rapidly become intractable as the number of atoms considered increases; therefore, approximations to these interactions have been developed in the form of empirical and semi-empirical potentials (Ortiz, 2001; Mishin, 1999) that can be used in a molecular dynamics (MD) analysis. Figure 3 shows the relationship between the lattice parameter and the atomic energy for fcc aluminum as determined by Mishin (Mishin, 1999). As seen in the figure, an equilibrium point corresponding to the lattice constant of approximately $0.406 \mathrm{~nm}$ corresponds to the minimum energy state. As the spacing between atoms deviates from this value, the

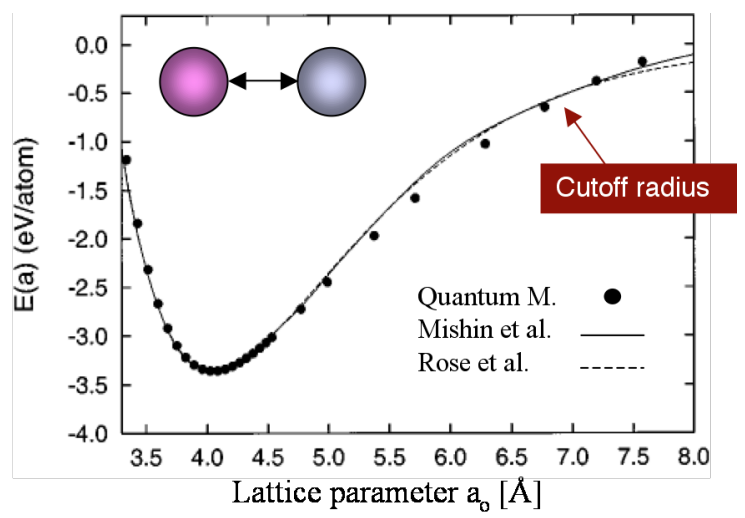

Figure 3: Energy per atom of fcc aluminum as a function of lattice parameter (adapted from Mishin, 1999) energy (and force) per atom increases rapidly and tends to return the atoms to their stable equilibrium positions.

Currently, interatomic potentials have been developed for many pure metals and some binary alloys. Many of the existing potentials have been developed with the goal of modeling a single type of phenomenon or property (e.g., phase transformations) and have dubious accuracy when they are used to predict other phenomena, hence, great care is needed in their selection and use. The development of new potentials, particularly for complex atomic systems (e.g., other binary alloys, ternary alloys, environmental effects), is an active area of research (e.g., Purja Pun, 2009; Apostol, 2010). 


\section{Molecular Dynamics Simulations of Fracture}

Based on the solution of Newton's equations of motion for a system of atoms interacting through a prescribed interatomic potential function, MD simulations are capable of determining the response of materials at atomic resolution. Although MD only approximates atomic interactions and considers very short time scales (typically on the order of nanoseconds), it is a powerful tool for understanding the underlying mechanisms of deformation and fracture. An important characteristic of MD simulations is that the operative deformation mechanisms emerge from the interatomic forces, and are not prescribed as input to the simulation model. Thus, not only can anticipated deformation processes be studied, but unanticipated ones may also be predicted. For example, a number of recent atomistic simulation studies on intergranular and transgranular crack propagation in aluminum have been published (Farkas, 2001; Hai, 2003; Yamakov, 2006; Warner, 2007; Warner 2009). The results of these investigations show that two main mechanisms of crack propagation operate and are highly dependent on crystallographic orientation, material type, external loading and temperature. The mechanisms are propagation through deformation twinning and propagation through the emission of full dislocations from the crack tip (see Figure 4). These results are largely consistent with well-established theoretical work (e.g., Rice, 1992; Rice, 1994; Tadmor, 2003).

Like any simulation method, MD simulations have inherent limitations and constraints that should always be considered in the interpretation of their results. Since current MD simulations typically address very short time scales $\left(\sim 10^{-9} \mathrm{~s}\right)$, very high stresses $\left(\sim 10^{9} \mathrm{~Pa}\right)$ and strain rates $\left(\sim 10^{7} \mathrm{~s}^{-1}\right)$ are required in order for atomic events to be observed. In an effort to determine whether or not it is possible for an MD-based method to predict the experimentally-determined mechanisms, Warner and Curtin used an extremely computationally efficient multiscale analysis method to perform simulations. They developed a unique simulation in which they considered a time range spanning six orders of magnitude - from picoseconds to microseconds. A transition in the operative mechanism from twinning to dislocation slip was found when the crack propagation rate was decreased to about one millionth of the rate that is often used in atomistic simulations (Warner, 2007). Nonetheless, the exact reasons why these length and time scales affect the propagation process are not completely understood. Additionally, the accelerated dynamics methods (Voter, 1997) and replica methods (Voter, 1999) that are needed to simulate relatively long time scales without adversely effecting predictions are under continued development.

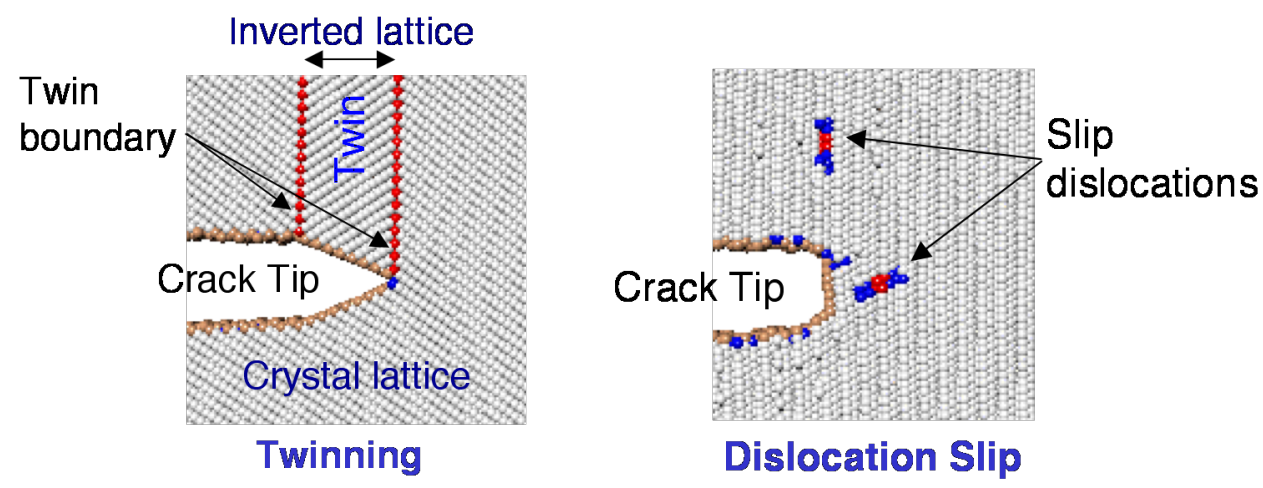

Figure 4: Twinning and slip near a crack tip (Yamakov, 2006)

\section{B. Multiscale Modeling}

Molecular dynamics simulations are limited not only by the volume of material and time scales that can be considered, but also by the types of boundary conditions and loadings that can be used in the analyses. The boundary conditions (e.g., periodic) and loadings (e.g., hydrostatic) that must be applied in MD simulations are often not representative of the state near a crack tip. Thus, a purely atomistic approach may not be practical for determining deformation and fracture in the vast majority of situations. One exception involves certain classes of nanocrystalline materials wherein the characteristic dimensions are extremely small; typical grain diameters in nanocrystalline metals range from about 5 to 50 nanometers (Schiotz, 1998). The promise that MD simulations are enabling to an unprecedented understanding of fundamental damage processes has motivated the development of multiscale modeling strategies that are needed to resolve many of the issues inherent to purely atomistic simulation. As a result, two broad classes of multiscale modeling strategies have been developed: concurrent multiscale modeling and sequential multiscale modeling. 


\section{Concurrent Multiscale Modeling}

Coupling atomistic and continuum simulations is particularly attractive for reducing computational cost in cases that require modeling of relatively large material domains to capture the complete deformation field, but where atomic refinement is needed only in very localized regions (e.g., near a crack tip or a dislocation core). By using coupled models, the size limitations of the atomistic simulation can be avoided by embedding an inner atomistic domain where complex dynamic processes and large deformation gradients exist within an outer domain where the deformation gradients are sufficiently small so that a continuum representation of the material becomes appropriate. Figure 5a is an illustration of a generalized concurrent multiscale model that couples MD to finite elements (FEM).

Over the past decade, various methods that couple material representations at different levels of refinement have been developed and offer significant computational advantages compared with purely MD simulations for predicting deformation and fracture processes (Curtin, 2003; Miller, 2002; Xiao, 2004; Shilkrot, 2002; Gumbsch, 1995; Rudd, 2005; Saether, 2009a). In these methods, the most challenging problem is the computational connection of the two different material representations at a prescribed interface where both the continuity of the material properties and the kinematics must be preserved during simulation. Continuity of material properties must be maintained while transitioning from individual atoms interacting through nonlocal forces to the local stress-strain field formalism of continuum mechanics.

Because of the requirement of directly coupling atoms in the MD domain to nodes in the FEM domain, most approaches to concurrent coupling are limited to two dimensions. Recently, an Embedded Statistical Coupling Method (ESCM, Figure 5b) that uses statistical averaging over both time and volume within atomistic subdomains (interface volume cells, IVC) at the MD-FEM interface has been developed and allows concurrent coupling in three dimensions (Saether, 2009a). In this method, nodal displacement boundary conditions are applied to the continuum FEM domain, which, in turn, generates interface reaction forces that are applied as constant traction boundary conditions to the atoms within the localized MD subdomain or IVC. The free MD surface, combined with surface volume cells (SVC), facilitates an accurate deformation state in the MD domain. The approach may be described as a local-nonlocal boundary value problem because it relates local continuum nodal quantities with nonlocal statistical averages of atomistic quantities over selected atomic subdomains. An iterative procedure between the MD statistical displacements and the FEM reaction forces ensures continuity at the interface. In this way, the problem of redefining continuum variables at the atomic scale seen in the direct coupling approaches is avoided, and the developed interface approach links different time and length scales between the MD and FEM domains.

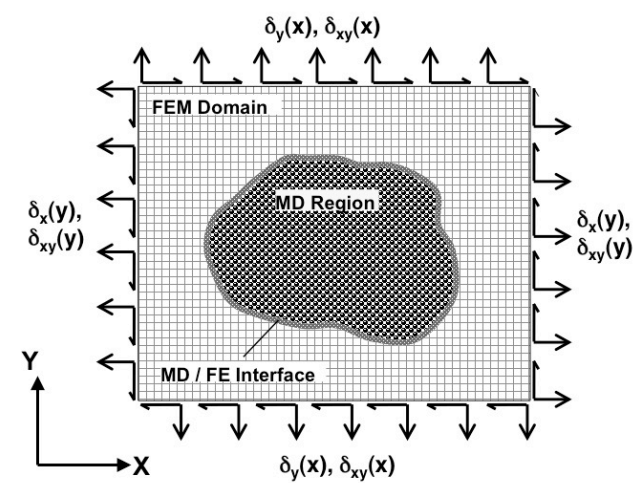

a) Embedded MD region within FE domain

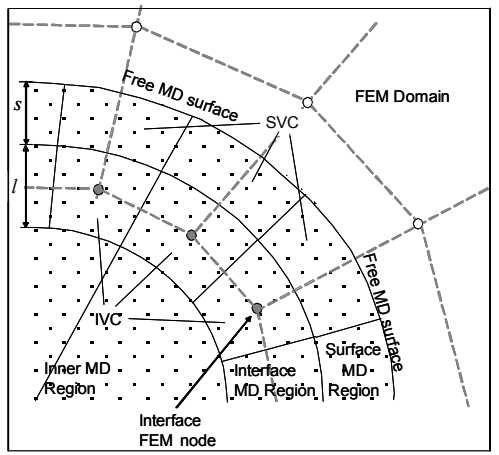

b) Structure of MD/FE interface

Figure 5: Model for concurrent MD-FEM coupling in ESCM (Saether, 2009a)

\section{Sequential Multiscale Modeling}

Concurrent multiscale models are only capable of interrogating very small and more importantly, very simple, domains. Even domains that have been considered using ESCM have very small out-of-plane dimensions, usually, not more than a few tens of nanometers. Thus, these analyses are only suitable for interrogating damage processes 
very near a crack tip in a single crystal or along a grain boundary. If larger, more complex domains that involve the fracture and deformation processes common to structural materials are to be considered, a sequential multiscale modeling strategy is needed. These modeling strategies may be based entirely on atomic-scale information (Glaessgen, 2006) or may include elements of larger-scale simulations, analytical solutions, experimentally determined behavior, or some combination thereof.

Sequential multiscale modeling, applied to either fracture process or deformation processes, involves some form of homogenization or averaging of the quantities of interest. The details of the mechanisms in the model at the lower length scale are passed as calibrated parameters to a separately analyzed model at the higher length scale. Because of the homogenization involved, various details are usually lost, thus, the form of the higher length scale model must be chosen carefully. A cohesive zone model (CZM) such as the one shown in Figure 6 may be used as the basis of homogenization in the fracture problem, thus, attempts to extract relevant parameters for the decohesion law of a CZM from atomistic simulations have been made by various groups. Recently, an approach based on the simulation of a dominant crack growing along a well-defined interface was proposed (Yamakov, 2006; Glaessgen, 2006).

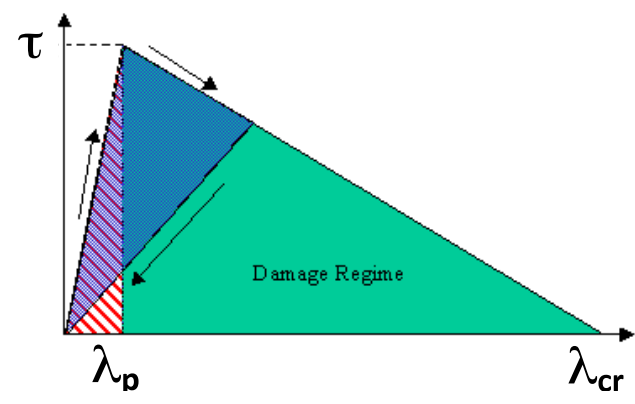

Figure 6: Constitutive relationship of a typical cohesive zone model
Here, an initial flaw was introduced within the atomistic simulation by screening the potential between atoms on opposite sides of the interface over a distance of a few nanometers. These works introduced the concept of a cohesive zone volume element (CZVE, see Figure 7) as a means of homogenizing the response of a small (nanometer scale) volume of atoms along the crack front. A statistically relevant traction displacement relationship was obtained from the CZVE and cast in the form of a continuum mechanics CZM. Thus, the CZVE, together with the MD simulation of fracture, enables the development of a (idealized) constitutive model of the near-crack tip fracture process that can be used in simulations at higher length scales (e.g., dislocation dynamics simulations and elasto-plastic microstructure simulations).

In the deformation problem, there is a natural, though currently unquantified, relationship between the plastic mechanisms that are determined by discrete simulations (e.g., molecular dynamics) and related continuum simulations (e.g., crystal plasticity). One natural linkage between these very different idealizations lies in examining the density of dislocations predicted using the discrete simulations and relating it to continuum quantities such as plastic slip. Determination of these types of relationships is another active area of research.

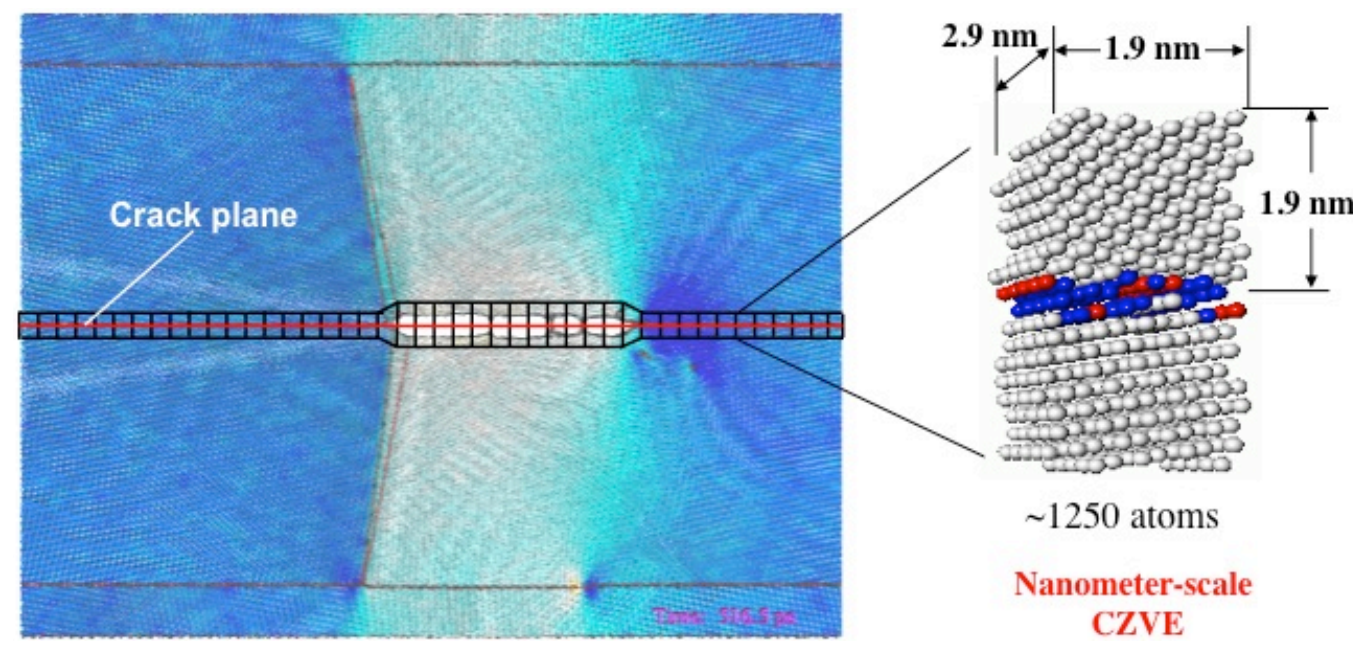

Figure 7: Cohesive Zone Volume Element (CZVE) and associated MD simulation (Yamakov, 2006) 
Several overviews of approaches for addressing various aspects of multiscale modeling have been published recently. An overview of concurrent multiscale modeling is given in Miller and Tadmor's compendium (Miller, 2009), a discussion of the use of cohesive zone models developed from molecular dynamics simulations is given in (Saether, 2009b) and a general discussion of concurrent multiscale modeling, sequential multiscale modeling and cohesive zone models is given in (Saether, 2009c).

\section{Mesoscale and Microscale Modeling}

Although some mechanisms of deformation and fracture must be interrogated using atomistic and related concurrent multiscale methods, many others may be best examined using mesoscale or microscale methods and related sequential multiscale models. Phenomena that may be addressed using these approaches include the effects of grain size, dislocation accumulation and interaction, cracking of second phase precipitates, and fatigue crack closure, on crack growth. Examples of relevant methods at the mesoscale and the microscale include several variants of plasticity theory and microstructure mechanics.

\section{Variants of Plasticity Theory}

Variants of plasticity theory that are of interest to Damage Science include discrete dislocation plasticity, strain gradient plasticity and crystal plasticity. At length scales on the order of a few microns or less, discrete dislocation plasticity can be used to simulate the generation, motion, accumulation and annihilation of individual dislocations and systems of dislocations in an otherwise elastic medium. At somewhat larger length scales (microns to tens of microns) where homogenization of the effects of dislocations is required but where gradient effects dominate plastic mechanisms near discontinuities, strain gradient plasticity can be used to describe plastic deformation near a crack tip or in the presence of small precipitates. Finally, at even larger length scales that are relevant to deformation of individual grains (tens of microns to hundreds of microns), crystal plasticity can be used to study plastic slip within a polycrystal that might contain tens, hundreds or even thousands of grains.

\section{Discrete Dislocation Plasticity}

Unlike continuum plasticity formulations wherein the elastic-plastic constitutive behavior is assumed, in discrete dislocation plasticity both the plastic stress-strain response and the corresponding evolution of the dislocation structures are predicted as part of the solution of the boundary value problem (Van der Giessen, 1995; Needleman, 2000; Cleveringa, 2000). Discrete dislocation (DD) plasticity simulations have been developed to represent large numbers of dislocations at relatively large length scales compared to atomic dimensions. In these approaches, dislocations are represented as lines of displacement discontinuity where the magnitude of the discontinuity is equal to the Burgers vector. Away from the dislocation core, the displacement, stress and strain fields may be suitably represented by analytic elasticity solutions. Simulations may involve infinite domains that are modeled using periodic boundary conditions or as finite domains with various applied boundary conditions.

Discrete dislocation plasticity is based on incrementally solving equations that describe the short- and long-range interactions between dislocations. During a simulation, the evolution of the dislocation field is obtained by forward integration of the governing equations so that the plastic stress-strain relationship is directly obtained during the analysis. As discussed by Van der Giessen and Needleman (Van der Giessen, 1995), the computation of the deformation history is performed in an incremental manner and involves determination of: (i) the Peach-Koehler forces on the dislocations; (ii) the rate of change of the dislocation structure caused by the motion of dislocations, the generation of new dislocations, their mutual annihilation, and their interaction with obstacles; (iii) the stress and strain state for the updated dislocation arrangement.

Discrete dislocation plasticity simulations may be performed in either two dimensions or in three dimensions. In two-dimensional simulations, dislocations are represented as point defects that are constrained to move along a particular slip plane. The result is a simplified representation that provides qualitative results of dislocation interaction and the resulting plastic and hardening behavior of material domains. While the inelastic stress-strain and hardening behavior is an outcome of the analysis, much investigation has been made to determine the formation of dislocation structures such as sub-cells, shear bands and low-angle grain boundaries (Devincre, 2001; Mughrabi, 1983; Thomson, 2002). These internal structures are related to the material plastic response and, due to constraints on dislocation mobility, result in hardening or increased toughness of the material. Other studies have been directed towards understanding the role of material length scales in fracture (Chakravarthy, 2010) and in comparing discrete 
dislocation simulations with continuum plasticity predictions (Saether, 2011). Figure 8a depicts a material domain containing a sharp crack subjected to Mode I loading. The initial nucleation of dislocations from the crack tip is shown in Figure $8 \mathrm{~b}$ and produces a pattern of maximum slip oriented along $\pm 60^{\circ}$ slip systems. This pattern, shown in Figure 8c, is consistent with continuum crystal plasticity predictions.

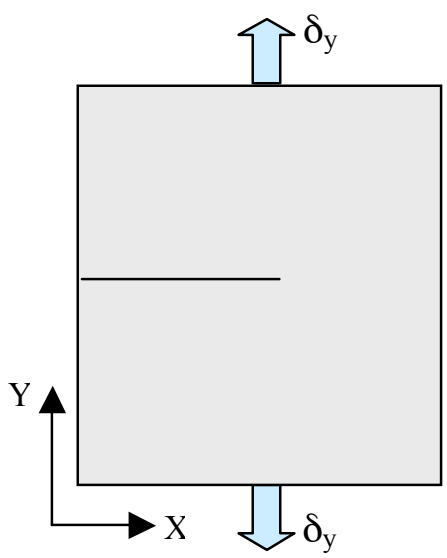

a) Sharp crack model

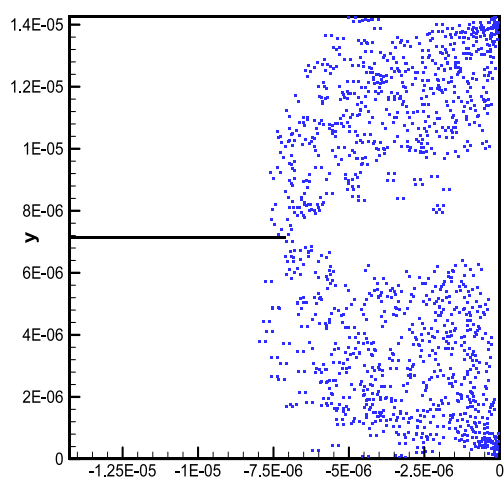

b) Dislocation generation

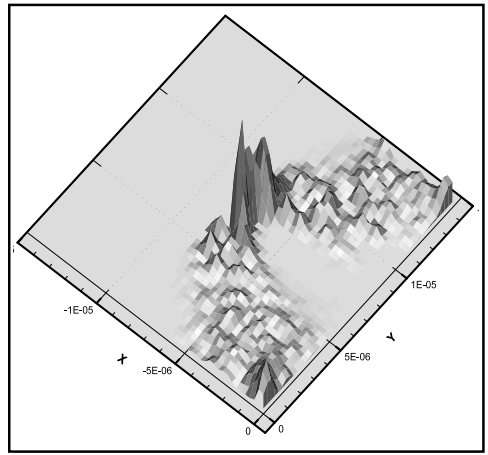

c) Profile of maximum slip

Figure 8: Simulation of dislocation nucleation near a crack tip under Mode I loading (Saether, 2011)

Three-dimensional modeling of dislocations promises to greatly reduce many of the approximations required in twodimensional DD, however, there is a tremendous increase in the complexity of the interactions and in the calculations required. Computational resources and modeling methods have been developed to account for full three-dimensional dislocation behavior but these methods are still under active development and are only beginning to be used outside of the groups that developed them. Among the most mature of the three-dimensional DD codes is the ParaDiS program (Arsenlis, 2007) that is being developed at Lawrence Livermore National Laboratory. Although the code cannot yet consider the effects of finite geometry on plasticity, it does account for threedimensional effects such as dislocation loops and also considers realistic long-range and short-range dislocation interactions.

Estimates of the strengths of obstacles and dislocation sources are important parameters in DD simulations because the interaction between dislocations and obstacles largely determines the strength and toughness of engineering alloys. Although efforts to understand dislocation-obstacle interactions span more than 60 years (e.g., Orowan, 1948), the use of atomistic simulations to attempt to quantify parameters such as obstacle strength are relatively new (e.g., Bacon, 2009). In Singh (Singh, 2011), the cutting of Guinier-Preston (GP) zones via cross-slip was modeled. GP zones are nanometer-scale monolayered disks that form during the early stages of age hardening and are common to many alloys including Al-Cu. Singh and co-workers considered the size of the GP zone as well as its orientation and offset relative to the dislocation. They found that the preferential interaction mechanisms and the resulting obstacle strength were strongly dependent on these parameters.

\section{Strain Gradient Plasticity}

Strain gradient plasticity is a class of continuum theories that assumes that any increase in flow strength or hardness of a material is caused by the generation and storage of geometrically necessary dislocations (GNDs) as is required to accommodate gradients of plastic shear strain such as those near a crack tip (Ashby, 1970; Nye, 1953; Fleck, 1993). Both GNDs and statistically stored dislocations (SSDs) are explicitly modeled by MD and DD methods; however, consideration of their effects in a homogenized, or continuum, formulation requires special treatment. While conventional continuum plasticity theories implicitly account for the generation and storage of SSDs, strain gradient plasticity theories are based on the observation that gradients of plastic deformation result in the generation and storage of GNDs as well. A basic tenant of these theories is that GNDs are produced by micron-scale gradients at a density comparable to, or greater than, that of SSDs, thus increasing the total dislocation density and the resistance to plastic flow. Thus, SSDs are associated with uniform deformation, while GNDs are associated with gradients as shown in Figure 9. 
One of the earliest characterizations of the effect of GNDs on material response was made by Fleck and coworkers in 1994 (Fleck, 1994). Fleck determined the tension and torsion responses of polycrystalline copper (99.99\%) wires for diameters in the range from $12 \mu \mathrm{m}$ to $170 \mu \mathrm{m}$. It was found that the wire diameter has very little effect on the true stress-strain response in tension for the five wire diameters considered. However, under torsion, the specimens demonstrated a profound hardening with decreasing wire diameter. If the constitutive response were independent of strain gradients, results for both tension and torsion loading would show no dependence on material dimensions. Dependence of material response on characteristic dimension (e.g, wire diameter) is indicative of the role of GNDs on strain gradient hardening. In addition to the work of Fleck on small diameter wires, many other experimental characterizations of the effect of strain gradients and the accompanying geometrically necessary dislocations have been undertaken. Some of these include the work of Stolken (Stolken, 1998) on bending of thin foils and various studies on nanoindentation (e.g., the work of Nix (Nix, 1998)).

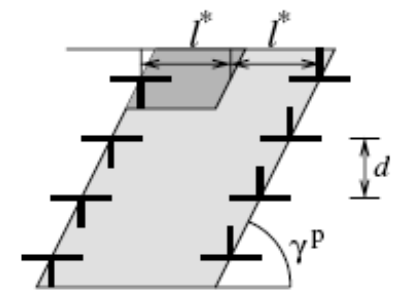

(a) Crystal strained by statistically stored dislocations

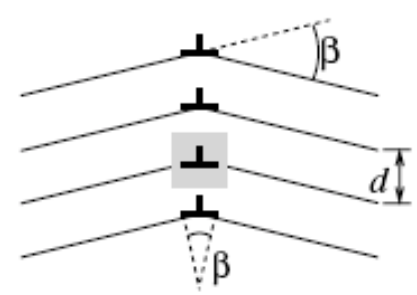

(b) Crystal strained by geometrically necessary dislocations

Figure 9: Uniform and gradient deformations associated with SSDs and GNDs, respectively (after Brinckmann, 2006)

From a microstructural point of view, there are several mechanisms or physical features that may determine the relative importance of various characteristic length scales. For plastic deformation, length scales that are related to dislocation structures, such as typical distances between dislocations and sizes of dislocation cells, are believed to be of greatest importance. However, irrespective of the physical feature considered, the number of dislocations within a homogenization volume must be sufficiently large and uniformly distributed such that meaningful averages can be taken, thus establishing an absolute lower bound on an effective length scale in these or any other continuum theories (Niordson, 2003).

\section{Crystal Plasticity}

At length scales where strain gradients are small, but where microstructural details of individual grains of material remain important, theories of crystal plasticity have been developed to predict plastic slip within an individual grain of a material (Taylor, 1925; Hill, 1972; Pierce, 1983) and for systems of polycrystals (Sarma, 2002). Plastic slip can be thought of as the homogenization of dislocation slip, in a continuum sense, on a slip plane in a slip direction. In crystal plasticity, Schmid's law is used to transform the stress from an arbitrary orientation into the orientation of each of the slip systems. This resolved shear stress, $\tau$, is given by

$$
\tau=\sigma \cdot n \cdot s=\sigma \cos \phi \cos \lambda
$$

where $\phi$ and $\lambda$ are the angles that the slip plane normal $\mathbf{n}$ and slip direction $\mathbf{s}$ make with the loading axis, respectively, as shown in Figure 10. Slip is assumed to occur once $\tau$ reaches a critical value. The term $\cos \phi \cos \lambda$ is called the Schmid factor.

The fundamental kinematical assumption of crystal plasticity is that the material flows by dislocation motion while the lattice itself undergoes elastic deformations and rotations (Asaro, 1977; Asaro, 1983). In other words, it is assumed that the total deformation, $F$, can be decomposed into plastic and elastic deformation as shown in Figure 11. The plastic deformation gradient, $F^{P}$, becomes nonzero when dislocations begin to move through the crystal 


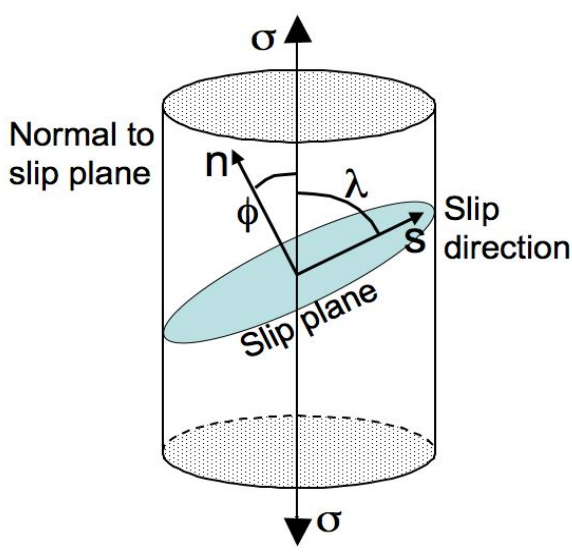

Figure 10: Resolution of applied stress on a slip system

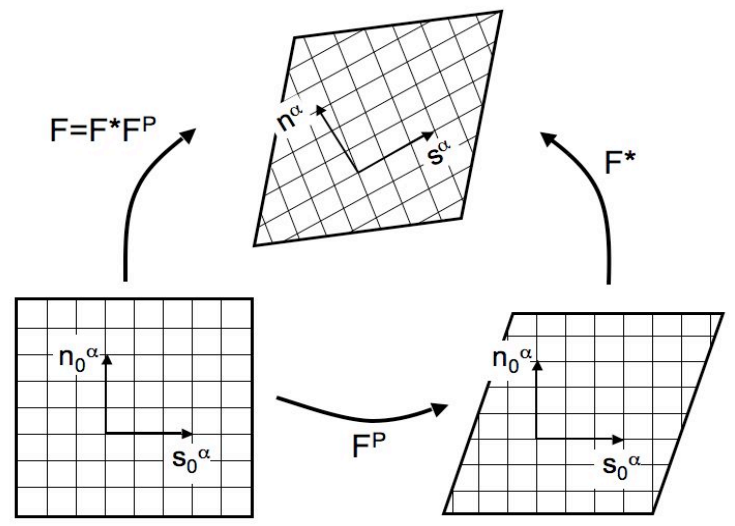

Figure 11: Decomposition of total deformation into $\mathrm{F}^{\mathrm{Y}}$ and $F^{*}$. (Asaro, 1977)

lattice. In addition to the plastic deformation, there is an elastic deformation gradient, $F^{*}$, wherein the lattice may be stretched, shortened or distorted, but that involves no relative movement between the material and the lattice.

Crystal plasticity formulations are being used to represent grain-scale material response in metallic microstructures under monotonic and cyclic loading. One recent combined experimental and computational study employed crystal plasticity within a finite element-based micromechanics model to study the effect of microstructure on fatigue crack nucleation in 7075-T651, an aluminum alloy that is known to initiate dominant fatigue cracks with the cracking of iron-bearing $\left(\mathrm{Al}_{7} \mathrm{Cu}_{2} \mathrm{Fe}\right)$ particles (Bozek, 2008; Hochhalter, 2010; Hochhalter, 2011). The experimental phase of the study showed that, under cyclic loading representative of flight conditions, a small percentage (1-10\%) of the particles in the alloy crack and only a few percent of those particles nucleate fatigue cracks into the surrounding microstructure.

In the computational phase of the study, both idealized and replicated (experimentally determined) microstructures were modeled. The idealized microstructures were used to develop finite element models that considered a semiellipsoidal surface particle surrounded by a single grain. Those models were used to determine the percentage of particles, as a function of parameterized shape and grain orientation, that were likely to crack. The replicated microstructures were used to develop finite element models (Figure 12a) that, together with crystal plasticity, explained the reasons why only a small percentage of the cracks in the particles extended into the surrounding grains. The study showed that cracks in the particles grow into the surrounding microstructure only when a critical combination of accumulated slip and local stress was reached. The field of accumulated slip is shown in Figure $12 \mathrm{~b}$. Based on the computed results, a model for predicting the number of cycles required to extend a crack beyond the particle-grain interface was developed.

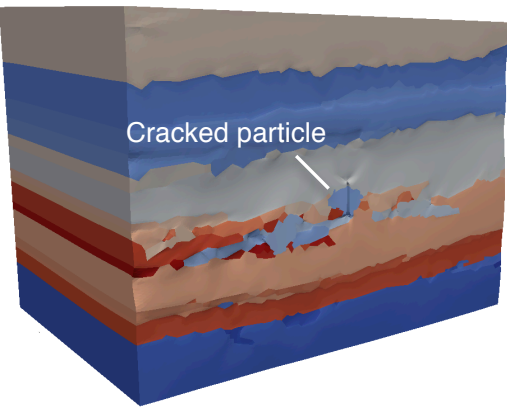

(a) Material morphology. Colors represent grains of different orientations.

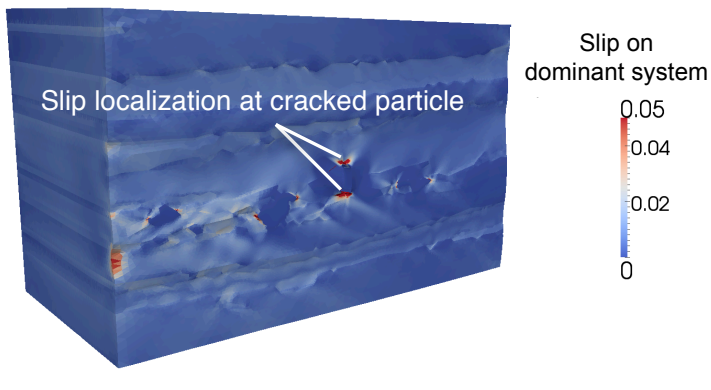

(b) Accumulated slip on the dominant system at $1 \%$ strain applied in the $x$-direction

Figure 12: Finite element model of an AA 7075-T651 polycrystal containing a cracked surface particle (Hochhalter, 2010; Hochhalter, 2011) 
There are several shortcomings of such microstructural studies, including the necessity of nonlocal analysis for computing crack driving forces, the incomplete representation of grain boundary mechanisms, and the lack of a rigorous model for crack propagation. Nonlocal analysis is required because a strain singularity of unknown order is present at a microstructurally-small crack. Grain boundary mechanisms, e.g., the passing of dislocations between grains, are not accounted for in conventional crystal plasticity formulations; instead, only a sudden change in the Schmid tensor across the grain boundary is represented. Recent work is aimed at developing and incorporating more rigorous local and nonlocal approaches for use in finite element models of crystal plastic behavior (Ma, 2006). Similarly, current grain-scale crack propagation models are ad hoc and rely on macroscale crack growth characterization for input; however, more rigorous grain-scale models are being developed based on multiscale modeling and in-situ experiments.

\section{Microstructure Modeling}

The generation of polycrystalline microstructures requires sophisticated modeling methods that are actively being developed (Raabe, 2001; Rollett, 2006; Brahme, 2006; Rollett, 2007). Among the most promising and realistic of the treatments of three-dimensional microstructure reconstruction is the work of Rollett and coworkers. In this approach, adopted in Hochhalter, 2010 and Hochhalter, 2011, a microstructural reconstruction is based on electron backscatter diffraction (EBSD) maps taken in two orthogonal cross sections of a sample of material: one perpendicular to the normal direction (ND) and the other perpendicular to the rolling direction (RD). The maps are in the form of $x, y$ coordinates of points throughout the cross sections and their corresponding crystallographic orientations. Grain boundaries are inferred at locations where a threshold value of misorientation is determined (Brahme, 2006). Once the microstructure and orientations are defined, discretization of the polycrystal into finite elements may proceed in the manner discussed in Veilleux (Veilleux, 2011).

\section{In-Situ Experiments}

High-resolution measurement techniques used in conjunction with mechanical testing can greatly increase the understanding of the complexities of damage under realistic conditions, help to validate some of the nano/microscale analysis results and provide input for microscale analyses. An experimental methodology based on a scanning electron microscope (SEM) equipped with an in-situ loading frame, video image correlation (VIC) system and EBSD system has been developed to characterize damage processes in metallic single crystals, bicrystals and polycrystals.

VIC is a non-contact method for obtaining full deformation and strain fields by tracking displacements on a speckled surface wherein the displacement and strain resolutions are largely determined by the size of the speckles and the available magnification. The method can measure large strains and accommodate significant rigid body movement

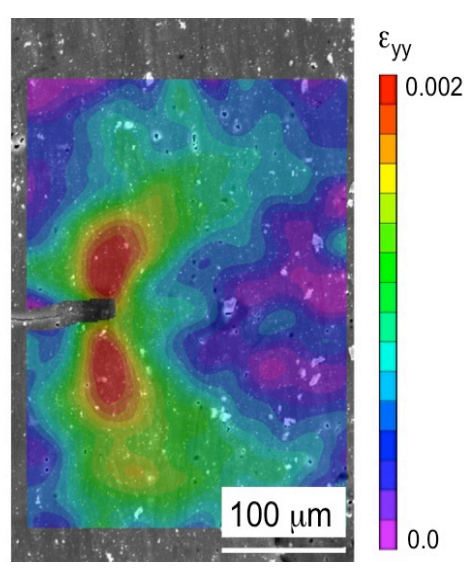

Figure 13: VIC image of strain field near a crack tip provided that the object does not leave the field of view of the camera. Figure 13 is a result from such an experiment where a fatigue-cracked, single-crystal of an aluminum alloy was loaded uniaxially in the $y$-direction and strains were measured using high-resolution VIC. E-beam lithography was used to deposit random speckle patterns (with speckle sizes of about 50 $\mathrm{nm}$ ) to obtain displacement measurements with a resolution of about $10 \mathrm{~nm}$. The VIC system used within the ESEM is based on VIC systems that have been used to interrogate displacement and strain fields at larger length scales (Sutton, 2009; Avril, 2008).

EBSD has been adapted to enable determination of the extent of plastic deformation near a fatigue crack tip and crack tip wake. Plasticity near the crack tip is related to plastic strain gradients and thus the GND density. Recent studies of single crystals and bicrystals (Sun, 2000; Kysar, 2002) have shown that it is possible to extract some of the components of the Nye dislocation density tensor (Nye, 1953) using orientation data obtained by EBSD mapping, provided that the crystal orientation and deformation conditions are carefully controlled to constrain the number of independent components. 
The approaches in Sun (Sun, 2000) and Kysar (Kysar, 2002) were adapted by Gupta (Gupta, 2009) to interrogate the EBSD orientation data obtained near a fatigue crack in a precipitation-hardened aluminum alloy, Al-Cu-Mg 2024T351. The intra-grain misorientation map (Figure 14a) displays changes in the local orientation, along with large amounts of intra-granular misorientation associated with the large plastic deformation in the vicinity of a crack tip wake. White regions in Figure 14a correspond to pixels that were not indexed. The misorientation map reveals distinctions in the morphology of plastic damage, e.g. the presence of slip-bands near the crack tip wake.

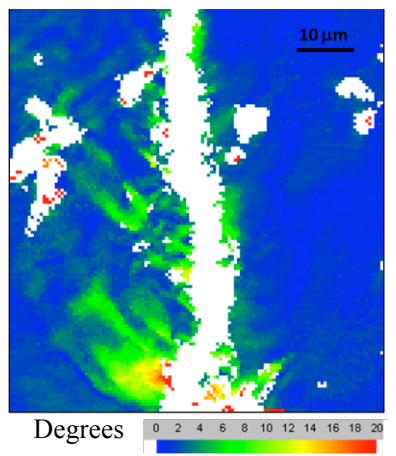

(a) EBSD misorientation map

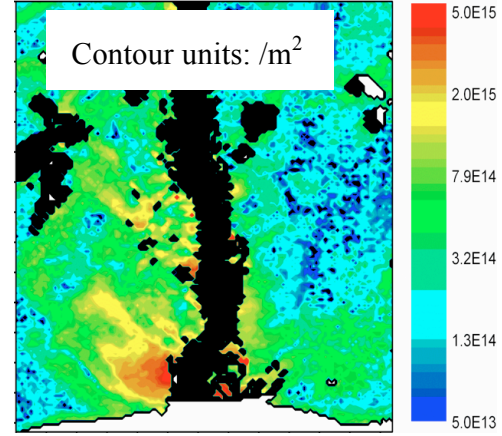

(b) Enhanced GND density map

Figure 14: Maps of misorientation and geometrically necessary dislocation density (Gupta, 2009)

Figure $14 \mathrm{~b}$ shows the estimated distribution of GND density within the scanned area. The regions of lower dislocation density (i.e., base material, $\sim 0.5-1 \times 10^{14} / \mathrm{m}^{2}$ ) are separated by regions of higher dislocation density (i.e., plastically-deformed crack-wake, $\geq 10^{15} / \mathrm{m}^{2}$ and higher), and can be identified by marked orientation change (Figure 14a) or by the enhanced dislocation density (Figure 14b). The boundaries of these banded structures depicting dislocation patterning contain a high GND density while regions within the bands are relatively free of dislocations that contribute to lattice curvature. An inhomogeneous distribution of the dislocation density becomes obvious for such cases.

The measurements of strain fields, local orientation changes and estimates of GND content near crack tips and wakes of fatigue cracks can be compared with those predicted by nanoscale and microscale computational models; however, the complexity of structural alloys encumbers the comparison. Rather, simple microstructures having prescribed crystallographic orientations may enable direct comparisons to be made. Single-crystals or bi-crystals of pure aluminum or simple two-component alloys may be developed using the Czochralski or Bridgman crystal growth methods and used in place of engineering alloys depending on the goals of the particular experimental study. The materials can be readily characterized for crystallographic orientation and grain structure, and mechanical test specimens can be machined to study various damage processes in specific crystallographic orientations. These relatively simple materials may be substantially closer in composition and structure to the idealizations considered by many of the analyses (e.g., MD, DD) and thus eliminate variables that complicate quantitative (and qualitative) comparison.

\section{Concluding Remarks}

Methods that are contributing to the analysis and characterization of fundamental deformation and fracture processes in metallic materials, including atomistic simulation, multiscale modeling, mesoscale and microscale modeling, and in-situ experiment, have been discussed. Each of the methods discussed herein has unique strengths, weaknesses and applicability; however, the most significant outcome of these techniques is not seen when the methods are implemented individually, but rather, when the methods are combined. For example, molecular dynamics simulations may be used to determine input parameters for discrete dislocation plasticity models of deformation and as the foundation for near-crack tip cohesive zone relationships. Similarly, discrete dislocation plasticity simulations may be used to determine values of the parameters needed for strain gradient plasticity simulations. As part of an integrated approach, emerging experimental methods may aid in an overall understanding of the complexities of damage under realistic conditions, help to validate some of the nano/micro-scale analysis results and provide input for microscale analyses. 
These and similar computational and experimental efforts are the basis for a bold paradigm shift in material science and mechanics of materials that will, for the first time, enable rigorous design and analysis of material microstructures and structural components having small characteristic dimensions. As the work described in this paper matures, it will reduce the dependence of material development on heuristic trial-and-error that results in production and evaluation of numerous prototypes of a material before a suitable one is found. Similarly, the work will reduce reliance on empiricism in engineering design and development, particularly for structures that are too small to be analyzed using current approaches. Many early steps have been taken to develop useful techniques for such analysis and characterization over a range of length scales. Several of them have been summarized in this paper.

\section{Acknowledgements}

The authors wish to thank their colleagues, funded over the past several years by the NASA Aviation Safety Program, for their many contributions to the Damage Science project, including Professors A.R. Ingraffea and D.H. Warner at Cornell University, W.A. Curtin at Brown University, Y. Mishin at George Mason University, R.G. Kelly and M. Neurock at the University of Virginia, M.A. Sutton at the University of South Carolina and Dr. H. Schreier at Correlated Solutions, Inc. Special thanks are due to Dr. R.S. Piascik who first envisioned Damage Science and recognized its benefits to NASA.

\section{References}

Apostol, 2010: Apostol, F. and Mishin, Y., "Angular-Dependent Interatomic Potential for the Aluminum-Hydrogen System," Phys. Rev. B, vol. 82, 2010, 144115.

Arsenlis, 2007: Arsenlis, A., Cai, W., Tang, M., Rhee, M., Oppelstrup, T., Hommes, G., Pierce, T.G., and Bulatov, V.V., "Enabling Strain Hardening Simulations with Dislocation Dynamics," Modelling Simul. Mater. Sci. Eng, vol. 15, 2007, pp. 553-595.

Asaro, 1977: Asaro, R.J. and Rice, J.R., "Strain Localization in Ductile Single Crystals," J. Mech. Phys. Solids, vol. 25, 1977, pp. 309-338.

Asaro, 1983: Asaro, R.J., “Crystal Plasticity,” J. Applied Mech, vol. 50, 1983, pp. 921-934.

Ashby, 1970: Ashby, M.F., “The Deformation of Plastically Non-Homogeneous Materials," Phi. Mag, vol. 21, 1970, pp. 399-424.

Avril, 2008: Avril, S., Pierron, F., Sutton, M.A. and Yan, J., "Identification of Elasto-Visco-Plastic Parameters and Characterization of Lüders Behavior Using Digital Image Correlation and the Virtual Fields Methods," Mech. of Matls, vol. 40, no. 9, 2008, pp. 729-742.

Bacon, 2009: Bacon, D.J. and Osetsky, Y.N., "Mechanisms of Hardening Due to Copper Precipitates in Iron," Philosophical Magazine, vol. 89, no. 34-36, 2009, pp. 3333-3349.

Bozek, 2008: Bozek, J.E., Hochhalter, J.D., Veilleux, M.G., Liu, M., Heber, G., Sintay, S.D., Rollett, A.D., Littlewood, D.J., Maniatty, A.M., Weiland, H., Christ, Jr., R.J., Payne, J., Welsh, G., Harlow, D.G., Wawrzynek, and P.A., Ingraffea, A.R., "A Geometric Approach to Modeling Microstructurally Small Fatigue Crack Formation: I. Probabilistic Simulation of Constituent Particle Cracking in AA 7075-T651," Modelling Simul. Mater. Sci. Eng, vol. 16, 2008.

Brahme, 2006: Brahme, A., Alvi, M.H., Saylor, D., Fridy, J., and Rollett, A.D., "3D Reconstruction of Microstructure in a Commercial Purity Aluminum," Scripta Mater, vol. 55, 2006, pp. 75-80.

Brinckmann, 2006: Brinckmann, S., Siegmund, T., and Huang, Y.-G., "A Dislocation Density Based Strain Gradient Model," Int. J. Plast, vol. 22, 2006, pp. 1784-1797.

Chakravarthy, 2010: Chakravarthy, S.S. and Curtin, W.A., "Origin of Plasticity Length-Scale Effects in Fracture," Phys. Rev. Lett, vol. 105, 2010.

Cleveringa, 2000: Cleveringa, H.M.M., Van der Giessen, E. and Needleman, A., "A Discrete Dislocation Analysis of Mode I Crack Growth,” J. Mech. Phys. Solids, vol. 48, 2000, pp. 1133-1157. 
Curtin, 2003: Curtin, W.A. and Miller, R.E., "Atomistic/Continuum Coupling in Computational Materials Science," Modelling Simul. Mater. Sci. Eng, vol. 11, 2003, pp. R33 - R68.

D’Agostino, 2005: D’Agostino, S.D., "Failure Analysis of MEMs Field Effect Transistor Support Membrane," Presentation at NASA Jet Propulsion Laboratory, 2005.

Devincre, 2001: Devincre, B., Kubin, L.P., Lemarchand, C., and Madec, R., "Mesoscopic Simulations of Plastic Deformation," Mat. Sci. Engrg., vol. A309-310, 2001, pp. 211-219.

Emery, 2009: Emery, J.M., Hochhalter, J.D., Wawrzynek, P.A., Heber, G. and Ingraffea, A.R., "DDSim: A Hierarchical, Probabilistic, Multiscale Damage and Simulation System - Part I: Methodology and Level I," Engng Fract Mech, 2009, doi:10.1016/j.engfracmech.2009.02.018.

Farkas, 2001: Farkas, D., Duranduru, M., Curtin, W. A., and Ribbens, C, "Multiple-Dislocation Emission from the Crack Tip in the Ductile Fracture of Al," Phil. Mag, vol. A 81, 2001, pp. 1241-1255.

Fleck, 1993: Fleck, N.A. and Hutchinson, J.W., "A Phenomenological Theory for Strain Gradient Effects in Plasticity," J. Mech. Phys. Solids, vol. 41, 1993, pp. 1825-1857.

Fleck, 1994: Fleck, N.A., Muller, G.M., Ashby, M.F. and Hutchinson, J.W., "Strain Gradient Plasticity: Theory and Experiment," Acta Metall. Mater, vol. 42, no. 2, pp. 475-487, 1994.

Glaessgen, 2006: Glaessgen, E.H., Saether, E., Phillips, D.R., and Yamakov, V., "Multi-Scale Modeling of GrainBoundary Fracture: Cohesive Zone Models Parameterized from Atomistic Simulations," Proceedings of the 47th AIAA/ASME/ASCE/AHS/ASC Structures, Structural Dynamics, and Materials Conference, AIAA-2006-1674-CP, AIAA, Newport, RI, May 1-4, 2006.

Gumbsch, 1995: Gumbsch, P. and Beltz, G.E., "On the Continuum Versus Atomistic Description of Dislocation Nucleation and Cleavage in Nickel," Modelling Simul. Mater. Sci. Eng, vol. 3, 1995, pp. 597-613.

Gupta, 2009: Gupta, V.K., Diffraction-Based Study of Fatigue Crack Initiation and Propagation in Aerospace Aluminum Alloys," University of Virginia, PhD Thesis, 2009.

Hai, 2003: Hai, S. and Tadmor, E. B., "Deformation Twinning at Aluminum Crack Tips," Acta Mater, vol. 51, 2003, pp. 117-131.

Hill, 1972: Hill, R. and Rice, J.R., "Constitutive Analysis of Elastic-Plastic Crystals at Arbitrary Strain," J. Mech. Phys. Solids, vol. 20, 1972, pp. 401-413.

Hochhalter, 2010: Hochhalter, J.D., Littlewood, D.J., Christ Jr., R.J., Veilleux, M.G., Bozek, J.E., Ingraffea, A.R., Maniatty, A.M., "A Geometric Approach to Modeling Microstructurally Small Fatigue Crack Formation: II. Physically Based Modeling of Microstructure-Dependent Slip Localization and Actuation of the Crack Nucleation Mechanism in AA 7075-T651," Modelling Simul. Mater. Sci. Eng, vol. 18, 2010.

Hochhalter, 2011: Hochhalter, J.D., Littlewood, D.J., Veilleux, M.G., Bozek, J.E., Maniatty, A.M, Rollett, A.D., and Ingraffea, A.R., "A Geometric Approach to Modeling Microstructurally Small Fatigue Crack Formation: III. Development of a Semi-Empirical Model for Nucleation," Modelling Simul. Mater. Sci. Eng, in review.

Kysar, 2002: Kysar, J.W. and Briant, C.L., "Crack Tip Deformation Fields in Ductile Single Crystals," Acta Mater, vol. 50, 2002, pp. 2367-2380.

Lee, 2002: Lee, Z., Rodriguez, R., Lavernia, E.J. and Nutt, S., "Microstructural Evolution of Cryomilled Nanocrystalline Al-Ti-Cu Alloy," Ultrafine Grained Materials II, Y.T. Zhu, T.G. Langdon, R.S. Mishra, S.L. Semiatin, M.J. Saran, and T.C. Lowe, Eds., TMS (The Minerals, Metals \& Materials Society), 2002.

Ma, 2006: Ma, A., Roters, F. and Raabe, D., "On the Consideration of Interactions Between Dislocations and Grain Boundaries in Crystal Plasticity Finite Element Modeling - Theory, Experiments, and Simulations," Acta Mater, vol. 54, 2006, pp. 2181-2194.

Miller, 2002: Miller, R.E. and Tadmor, E.B., "The Quasicontinuum Method: Overview, Applications and Current Directions," J. Comp. Aided Mat. Design, vol. 9, 2002, pp. 203-239.

Miller, 2009: Miller, R.E. and Tadmor, E.B., "A Unified Framework and Performance Benchmark of Fourteen Multiscale Atomistic/Continuum Coupling Methods,” Modelling Simul. Mater. Sci. Eng, vol. 17, 2009.

14

American Institute of Aeronautics and Astronautics 
Mishin, 1999: Mishin, Y., Farkas, D., Mehl, M. J., and Papaconstantopoulos, D. A., "Interatomic Potentials for Monoatomic Metals from Experimental Data and ab initio Calculations," Phys. Rev. B, vol. 59, 1999, pp. 33933407.

Mughrabi, 1983: Mughrabi, H., "Dislocation Wall and Cell Structures and Long-Range Internal Stresses in Deformed Metal Crystals,” Acta Metall, vol. 31, 1983, pp. 1367-1379.

Nair, 2010: Nair, A.K., Warner, D.H., Hennig, R.G. and Curtin, W.A., "Coupling Quantum and Continuum Scales to Predict Crack Tip Dislocation Nucleation," Scripta Mat, vol. 63, no. 12, 2010, pp. 1212-1215.

Needleman, 2000: Needleman, A., “Computational Mechanics at the Mesoscale," Acta Mater, vol. 48, 2000, pp. 105-124.

Needleman, 2001: Needleman, A. and Van der Giessen, E., "Micromechanics of Fracture: Connecting Physics to Engineering," MRS Bulletin, March 2001, pp. 211-214.

Niordsen, 2003: Niordson, C.F., and Hutchinson, J.W., “On Lower Order Strain Gradient Plasticity Theories,” Euro. J. Mech. A/Solids, vol. 22, 2003, pp. 771-778.

Nix, 1998: Nix, W.D. and Gao, H., "Indentation Size Effects in Crystalline Materials: A Law for Strain Gradient Plasticity," J. Mech. Phys. Solids, vol. 46, 1998, pp. 411-425.

Nye, 1953: Nye, J.F., "Some Geometrical Relations in Dislocated Crystals,” Acta Metal, vol. 1, 1953, pp. $153-162$.

Orowan, 1948: Orowan, E., Symposium on Internal Stresses in Metals and Alloys, Institute of Metals, London, 1948.

Ortiz, 2001: Ortiz, M., Cuitino, A.M., Knap, J. and Koslowski, M., "Mixed Atomistic-Continuum Models of Material Behavior: The Art of Transcending Atomistics and Informing Continua," MRS Bulletin, March 2001, pp. 216-221.

Pierce, 1983: Pierce, D., Asaro, R.J., and Needleman, A., "Material Rate Dependence and Localized Deformation in Crystalline Solids," Acta Metal, vol. 31, no. 12, 1983, pp. 1951-1976.

Purja Pun, 2009: Purja Pun, G.P. and Mishin, Y., "Development of an Interatomic Potential for the Ni-Al System," Phil. Mag, vol. 89, no. 34, 2009, pp. 3245-3267.

Raabe, 1998: Raabe, D., Computational Materials Science: The Simulation of Materials Microstructures and Properties, Wiley-VCH, Weinheim, 1998.

Raabe, 2001: Raabe, D., Sachtleber, M., Zhao, Z., Roters, F. and Zaefferer, S., "Micromechanical and Macromechanical Effects in Grain Scale Polycrystal Plasticity Experimentation and Simulation," Acta Mater, vol. 49, 2001, pp. 3433-3441.

Rice, 1992: Rice, J. R., “Dislocation Nucleation From a Crack Tip: An Analysis Based on the Peierls Concept,” J. Mech. Phys. Solids, vol. 40, 1992, pp. 239-271.

Rice, 1994: Rice, J.R. and Beltz, G.E., "The Activation Energy for Dislocation Nucleation at a Crack," J. Mech. Phys. Solids, vol. 42, 1994, pp. 333-360.

Rollett, 2006: Rollett, A.D., Campman, R. and Saylor, D., "Three Dimensional Microstructures: Statistical Analysis of Second Phase Particles in AA 70575-T651," Proceedings of the $10^{\text {th }}$ International Conference on Aluminum Alloys, vol. 519-521, 2006, pp. 1-10.

Rollett, 2007: Rollett, A.D., Lee, S.-B., Campman, R. and Rohrer, G.S., "Three-Dimensional Characterization of Microstructure by Electron Back-Scatter Diffraction,” Annu. Rev. Mater. Res, vol. 37, 2007, pp. $627-658$.

Rudd, 2005: Rudd, R.E., and Broughton, J.Q., "Coarse-Grained Molecular Dynamics and the Atomic Limit of Finite Elements,” Phys. Rev. B, vol. 58, 2005, pp. R5893-R5896.

Saether, 2009a: Saether, E., Yamakov, V. and Glaessgen, E.H., “An Embedded Statistical Method for Coupling Molecular Dynamics and Finite Element Analysis,” Int. J. Num. Meth. Engng, vol. 78, 2009, pp. 1292-1319.

Saether, 2009b: Saether, E. and Glaessgen, E.H., "The Development of Directional Decohesion Finite Elements for Multiscale Failure Analysis of Metallic Polycrystals," NASA/TM-2009-215715, 2009.

15

American Institute of Aeronautics and Astronautics 
Saether, 2009c: Saether, E., Yamakov, V. Phillips, D.R and Glaessgen, E.H., "An Overview of the State of the Art in Atomistic and Multiscale Simulation of Fracture," NASA/TM-2009-215564, 2009.

Saether, 2011: Saether, E., unpublished work, 2011.

Sarma, 2002: Sarma, G.B., Radhakrishnan, B. and Dawson, P.R., "Mesoscale Modeling of Microstructure and Texture Evolution During Deformation Processing of Metals," Adv Engng Mat, vol. 4, 2002, pp. 509-514.

Schiotz, 1998: Schiotz, J., Di Tolla, F.D. and Jacobsen, K.W., "Softening of Nanocrystalline Metals at Very Small Grain Sizes," Nature, vol. 391, 1998, pp. 561-563.

Shilkrot, 2002: Shilkrot, L.E., Miller, R.E., and Curtin, W.A., "Coupled Atomistic and Discrete Dislocation Plasticity," Phys. Rev. Lett, vol. 89, 2002, p. 025501-1.

Singh, 2011: Singh, C.V., Mateos, A.J. and Warner, D.H., "Atomistic Simulations of Dislocation-Precipitate Interactions Emphasize Importance of Cross-Slip,” Scripta Mat, vol. 64, 2011, pp. 398-401.

Stolken, 1998: Stolken, J.S. and Evans, A.G., "A Microbend Test Method for Measuring the Plasticity Length Scale," Acta Mater, vol. 46, 1998, pp. 5109-5115.

Sun, 2000: Sun, S., Adams, B.L. and King, W.E., "Observations of Lattice Curvature Near the Interface of a Deformed Aluminum Crystal," Phil Mag A, vol. 80, no. 1, 2000, pp. 9-25.

Sutton, 2009: Sutton, M.A., Orteu, J.-J. and Schreier, H.W., Image Correlation for Shape, Motion and Deformation Measurements: Basic Concepts, Theory and Applications, Springer, New York, NY, 2009.

Tadmor, 2003: Tadmor, E.B. and Hai, S., "A Peierls Criterion for the Onset of Deformation Twinning at a Crack Tip,” J. Mech. Phys. Solids, vol. 51, 2003, pp. 765-793.

Taylor, 1925: Taylor, G.I. and Elam, C.F., "The Plastic Extension and Fracture of Aluminum Crystals," Proc. Royal Soc. London A, vol. 108, 1925, pp. 28-51.

Thomson, 2002: Thomson, R., Levine, L.E., Shim, Y., Savage, M.F., and Dramer, D.E., "A Multi-Scale Theoretical Scheme for Metal Deformation," Comp. Model. Eng. Sci., vol. 3, 2002, pp. 245-253.

Van der Giessen, 1995: Van der Giessen, E. and Needleman, A., "Discrete Dislocation Plasticity: A Simple Planar Model," Modelling Simul. Mater. Sci. Eng, vol. 3, 1995, pp. 689-735.

Van der Giessen, 2002: Van der Giessen, E. and Needleman, A., "Micromechanics Simulations of Fracture," Ann. Rev. Mat. Res, 2002, pp. 141-162.

Veilleux, 2011: Veilleux, M.G., "Geometrically Explicit Finite Element Modeling of AA7075-T651 Microstructure with Fatigue Cracks," Cornell University, PhD Thesis, 2011.

Voter, 1997: Voter, A.F., "Hyperdynamics: Accelerated Molecular Dynamics of Infrequent Events," Phys. Rev. Lett, vol. 78, 1997, pp. 3908-3911.

Voter, 1999: Voter, A.F. and Sorensen, M.R., "Accelerating Atomistic Simulations of Defect Dynamics: Hyperdynamics, Parallel Replica Dynamics, and Temperature-Accelerated Dynamics," Materials Research Society Symposium - Proceedings, vol. 538, 1999, pp. 427-439.

Warner, 2007: Warner, D. H., Curtin, W. A., and Qu, S., "Rate Dependence of Crack-Tip Processes Predicts Twinning Trends in f.c.c. Metals," Nature Mater, vol. 6, 2007, pp. 876-880.

Warner, 2009: Warner, D. H. and Curtin, W. A., "Origins and Implications of Temperature-Dependent Activation Energy Barriers for Dislocation Nucleation in Face-Centered Cubic Metals," Acta Mater, vol. 57, 2009, pp. 42674277.

Xiao, 2004: Xiao, S.P., and Belytschko, T., "A Bridging Domain Method for Coupling Continua with Molecular Dynamics," Comput. Methods Appl. Mech. Engrg, vol. 193, 2004, pp. 1645-1669.

Yamakov, 2006: Yamakov, V., Saether, E., Phillips, D.R. and Glaessgen, E.H., “Asymmetric Inter-granular Crack Growth Along $\Sigma 99$ Grain Boundary in Aluminum by Molecular Dynamics and Finite Element Simulations," $J$. Mech. Phys. Solids, vol. 54, pp. 1899-1928, 2006.

16

American Institute of Aeronautics and Astronautics 